\title{
Characterization of Acacia mangium polyflavonoid tannins by MALDI-TOF mass spectrometry and CP-MAS 13C NMR
}

\begin{abstract}
The MALDI-TOF mass spectrometry (MS) and solid state CP-MAS 13C Nuclear Magnetic Resonance (NMR) spectroscopic technique were introduced to characterize Acacia mangium tannin (condensed tannins). The MALDI-TOF MS illustrated a series of peaks corresponding to oligomers of condensed tannins of up to 11 flavonoid units (3200 Da). A. mangium condensed tannins were found to consist predominantly of prorobinetinidin combined with profisetinidin and prodelphinidin. Both the MALDI-TOF mass spectra and the solid state CPMAS 13C NMR indicated that the A. mangium tannins obtained from Kudat, had an almost completely linear structure; In addition, Lembah Beringin, consist of "angular" polymer structure; and Tawau, has included "twice-angular" polymer structures present in oligomers type of up to 7 flavonoid units. The high degree of polymerization of linear, angular type, twice-angular structures and longer oligomer (3200 Da) chains have not been observed in previous studies of condensed tannins. The spectra also indicated that A. mangium tannins are more heavily branched and have higher degree of polymerization (>7.0) compared to commercial mimosa (A. mearnsii) tannin (4.9). Because tannins are phenolic, it was expected that they can be used to replace phenol-formaldehyde (PF) adhesives.
\end{abstract}

Keyword: MALDI-TOF; Polyflavonoid; Tannin; Polymer; CP-MAS 13C NMR; Acacia mangium 